\title{
ORIGINAL ARTICLE \\ Conjugated linoleic acid ameliorates hepatic steatosis by modulating intestinal permeability and gut microbiota in ob/ob mice
}

\author{
Shengli Gao', Yingying He², Liping Zhang',3, Lina Liu, Changfeng Qu 2,4, Zhou Zheng2,4 \\ and Jinlai Miao $2,3,4,5 *$ \\ 'Biomedical Center, Qingdao Medical College, Qingdao University, Qingdao, China; ${ }^{2}$ Key Laboratory of Marine Eco- \\ Environmental Science and Technology, First Institute of Oceanography, Ministry of Natural Resource, Qingdao, China; \\ ${ }^{3}$ Department of Special Medicine, School of Basic Medicine, Qingdao University; ${ }^{4}$ Qingdao Key Laboratory of Marine \\ Natural Products Research and Development, Qingdao, China; ${ }^{5}$ Guangxi Academy of Sciences, Nanning, China
}

\section{Popular scientific summary}

- The effect of highly purified conjugated linoleic acid (CLA) on lipid metabolism was examined in wild-type and ob/ob mice.

- CLA supplementation potentially contributed to ameliorating the liver inflammatory state and fat accumulation in ob/ob mice.

- CLA administration partly reversed the increased intestinal permeability in ob/ob mice.

- CLA consumption led to changes in gut microbiome composition.

- These results could provide a new perspective on the role of dietary CLA in hepatic fat accumulation.

Abstract

Background: Conjugated linoleic acid (CLA) is an effective supplement for reducing fat mass, but its effect on hepatic steatosis remains controversial.

Objective: This study aims to evaluate the effect of CLA on liver fat accumulation, inflammation, gut microbiome, and intestinal barrier integrity.

Design: Wild-type (WT) mice and ob/ob (OB) mice were randomly divided into four groups according to the treatment with/without 1\% CLA: WT, WT mice treated with CLA (WT-CLA), OB, and OB mice treated with CLA (OB-CLA). Lipid metabolism and hepatic fat accumulation were evaluated by changes in histological and biochemical parameters. Gene expressions related to liver inflammation and intestinal barrier integrity were examined. The effect of CLA on the gut microbiota population was investigated.

Results: The body weight, fatty tissue mass, and serum lipid levels of the WT-CLA group and OB-CLA group were separately lower than those of the WT group and OB group, but the livers of the WT-CLA group had more fatty lipids, higher triglyceride properties, and saturated fatty acid (FA) composition than those of the WT group, which was contrary to the effect of CLA on OB mice. Real time quantitative PCR results showed that CLA increased hepatic inflammation and intestinal permeability in the WT mice, while it significantly decreased the mRNA expression of liver TNF- $\alpha$, IFN- $\gamma$, and IL- $1 \beta$ and markedly ameliorated intestinal tight junction proteins in the $\mathrm{OB}$ mice. The gut microbiota testing indicated a higher abundance of beneficial bacteria (e.g., Lachnoclostridium, Roseburia, Dubosiella, Oscillibacter, and Anaerostipes) and a lower abundance of pro-inflammatory bacteria (e.g., Tyzzerella and Alistipes) in the OB-CLA group than those of the OB group. Correlation analysis suggested that gut microbiota correlated with liver inflammation, intestinal permeability, and hepatic FA composition.

Conclusion: CLA potentially contributed to ameliorating hepatic steatosis in OB mice via modulating liver inflammation, intestinal permeability, and gut microbiota, which suggests CLA is more suitable for people with obesity or overweight.

Keywords: conjugated linoleic acid; obesity; hepatic steatosis; intestinal permeability; gut microbiota 
$\mathrm{C}$ onjugated linoleic acid (CLA) is a family of polyunsaturated fatty acids (FAs) characterized by two conjugated double bonds in different geometric (cis- or trans-) and positional sites. Of the 28 known isomers of CLA, cis-9, trans-11-CLA (c9t11-CLA), and trans-10, cis-12-CLA (t10c12-CLA) are the most abundant isomers in natural and synthetic CLAs (1). With an amazing effect on decreasing body weight through fat mass reduction, CLA has been widely used by obese people to lose weight (2). Nevertheless, CLA has been found to promote hepatic steatosis in rodent animals, which might be a potential threat to human health $(3,4)$. Some studies indicated that the potential mechanisms of hepatic steatosis induced by CLA were insulin resistance and consequent hyperinsulinemia, which was closely associated with decreased adipokine (leptin) (5-7). However, surprisingly, CLA alleviated hepatic steatosis in leptin-deficient ob/ob mice, which was reversed by the presence of leptin (8). Therefore, undefined mechanisms might be involved in hepatic steatosis induced by CLA.

The liver receives venous blood from the intestine, stomach, and pancreas via the portal vein, which contains nutrients and metabolites of host and microbiome origin (9). Through this route, enterically derived substances, such as lipopolysaccharide (LPS), may affect liver function and cause steatohepatitis by triggering Toll-like receptor 4 (TLR4)-dependent injury in the liver (10-12), which could be restrained by high-density lipoprotein (HDL) (13). CLA supplementation was found to modulate inflammatory cytokines, maintain the mucosal barrier, and revert microbiota changes in the intestines of mice with Dextran sulfate sodium (DSS)-induced colitis (14). Consequently, we speculate that CLA might play different roles in regulating the intestinal structure and gut microbiota in normal and ob/ob mice.

In this study, the effects of CLA on obesity and correlated metabolic disorders were investigated in ob/ob and normal mice. We studied changes in intestinal permeability and the microbiome induced by CLA. By comparing the differences between the ob/ob and normal mice, we aimed to delineate the possible mechanisms of hepatic steatosis induced by CLA.

\section{Materials and methods}

\section{Reagents}

The commercial CLA mixture was purchased from Qingdao Auhai Biotech Co., LTD (Shandong, China). SP2560 Capillary GC Column (24056) was purchased from Merck KGaA (Darmstadt, Germany). Orlistat (ORL) was purchased from Cayman Chemical Co., Inc. (MI, USA). All serum biochemical test kits were purchased from the Nanjing Jiancheng Bioengineering Research Institute (Nanjing, China). All primers were obtained from Sangon
Biotech (Shanghai, China). The PureLink RNA Mini Kit (12183018A), high-capacity cDNA Reverse Transcription Kit (4368814), DyNAmo ColorFlash SYBR Green qPCR Kit (F416S), Gene JETTM Gel Extraction Kit (K0692), and Ion Plus Fragment Library Kit (4471252) were purchased from Thermo Fisher Scientific (CA, USA). The QIAamp DNA Stool Mini Kit (Cat: 51504) was purchased from Qiagen (Hilden, Germany). The Phusion Flash High-Fidelity PCR Master Mix (F548S) was purchased from NEB (MA, USA). Male leptin-deficient ob/ob mice (T001461) and C57BL/6J wild-type mice (N000014) were purchased from Shanghai Biomodel Organism Science \& Technology Development Company (Shanghai, China). The standard rodent diet (AIN93) was purchased from Qingdao Darenfucheng Animal Technology Co., Ltd. (Qingdao, China).

\section{CLA purification and gas chromatography analysis}

The commercial CLA mixture was purified with ureainclusion crystallization according to the Kim's method (15). Briefly, the urea-saturated methanol solution was heated to $70^{\circ} \mathrm{C}$ for dissolving the CLA mixture and cooled down to room temperature in a cooling water bath. The obtained solution was then filtered (pore size: $0.45 \mu \mathrm{m}$ ) to remove urea-inclusion compounds, which contained most of the saturated FAs and monoenoic acids. The filtrate was added to the $\mathrm{C}$ urea-saturated methanol solution at $70^{\circ} \mathrm{C}$. After the solution cooled to room temperature, CLA and urea formed CLA-urea-inclusion compounds, which were recovered when the solution mainly containing $\alpha$-linolenic acid was filtered off. The compounds were solved with distilled water, and the highly purified CLA was mainly in the upper layer. Hexane extraction was used to recover CLA, which was prepared after being washed three times with distilled water. The purified CLA was analyzed by gas chromatography (GC) according to the protocol of the SP-2560 capillary GC column (16). The contents of different CLA isomers were calculated using the area normalization method.

\section{Animals and experimental design}

Male leptin-deficient ob/ob mice (OB) and C57BL/6J wildtype mice (WT) aged 6 weeks were housed $4 /$ cage at $23^{\circ} \mathrm{C}$, with a $12 \mathrm{~h}$ light/12 h dark cycle and with a standard rodent diet and water ad libitum. After 1 week of adaptation, 20 WT mice and 20 OB mice were randomly divided into four groups as follows $(n=10)$ : WT (olive oil, $1.5 \%$ body weight), WT-CLA (CLA, 1.5\% body weight), OB (olive oil, $1.5 \%$ body weight), and OB-CLA (CLA, 1.5\% body weight). Olive oil was used as a vehicle because of the similar uptake and metabolite distribution of CLAs and oleic acid (17). The CLA and the olive oil were administered by oral gavage at 9:00 am every day for 5 consecutive weeks. The body weight, 24-h food intake, and body length (from 
nose to anus) were recorded weekly. The Lee index of obesity was calculated as follows: the cube root of body weight (g) was divided by naso-anal length $(\mathrm{cm})$ and multiplied by 1,000 (18). At the end of the experiment, each mouse was transferred to an empty cage to collect feces samples. The plasma was obtained from the retro-orbital plexus and centrifuged $\left(4,000 \mathrm{rpm}, 4^{\circ} \mathrm{C}, 40 \mathrm{~min}\right)$ to collect the serum samples. Fat tissue, liver, and colon were rapidly striped, weighed, and photographed. The feces, serum, and colon samples were stored at $-80^{\circ} \mathrm{C}$, and the adipose and liver tissues were fixed in $4 \%$ paraformaldehyde (PFA) for histological staining. All animal procedures complied with the China's 109 Regulation on the Use and Care of Laboratory Animals and were approved by the Ethics Committee of Laboratory Animal Care of Qingdao University.

\section{Histological staining and cell size calculation}

PFA-fixed fat and liver tissue sections were routinely stained with hematoxylin and eosin (H\&E) (19). After dehydration and transparency, the samples were embedded in paraffin and cut into 7- $\mu \mathrm{m}$-thick sections. Following deparaffinization and hydration, the sections were stained with H\&E Y solution. After the tissue slides were prepared, the images were visualized using a $\mathrm{BX} 63 \mathrm{~F}$ microscope and captured with a DP80 digital camera (Olympus, Tokyo, Japan) (20).

Using ImageJ software (http://imagej.nih.gov/ij/), the cell size was calculated $(19,21)$. First, a known scale from the BX63F microscope was used to calibrate ImageJ. The photos were then opened and converted into gray-scale images in the software. The removal of background noise and unwanted particles significantly improved the clarity of these images. The membrane structure and blank space of the cells were identified with gray-scale threshold values. Lastly, the cell size was calculated using the free 'Measure and Label Macro' plugin for ImageJ (http://rsbweb.nih.gov/ij/plugins/measure-label.html) and displayed as the cell surface area $\left(\mu \mathrm{m}^{2}\right)$. The shape parameter for cell selection was fixed between 0.35 and 1 (' 0 ' refers to a straight line, while ' 1 ' refers to a perfect circle). Cells touching the border of an image or shrunken cells were not counted. In every section, at least 200 adipocytes and 800 hepatocytes were used for the data analysis.

\section{Serum and liver lipid analysis}

The levels of serum total cholesterol (TG) and triglyceride (TC) were measured using a TG or TC Assay Kit (glycerol 3 phosphate oxydase and phenol 4-aminoantipyrine peroxidase Method). The serum level of free fatty acid (FFA) was tested with an enzyme linked immunosorbent assay (ELISA) commercial kit. All operations were carried out according to the manufacturer's instructions.

Total liver lipids were extracted using a mixture of methanol and chloroform $(1: 2, \mathrm{vol} / \mathrm{vol})$ and resolved in a mixture of hexane: diethyl ether: acetic acid (70: $30: 0.2$, by vol) for quantitative lipid class analyses in the thin-layer chromatography flame ionization detection system as previously described (22). Phospholipids (PL) were separated into classes by high-performance liquid chromatography (23). The FAs from the total PL, PL classes, cholesteryl esters (CE), and TG were esterified using sodium methylate and boron trifluoride, and quantitative data were analyzed using gas-liquid chromatography (24).

\section{Real-time quantitative PCR}

Total RNA was extracted using a PureLink RNA Mini Kit, and cDNA synthesis was performed using a high-capacity cDNA Reverse Transcription Kit. Each reverse transcription PCR (RT-PCR) was carried out with a diluted cDNA template, forward and reverse primers $(5 \mu \mathrm{m}$ each $)$, and a DyNAmo ColorFlash SYBR Green qPCR Kit (25). Real-time PCR was performed using the Applied Biosystems 7500 Fast Real-Time PCR System (Thermo Fisher Scientific, CA, USA). Primer sequences were as follows: TNF- $\alpha$ forward primer 5'-TAG CCA GGA GGG AGA ACA GA-3', reverse primer 5'-TTT TCT GGA GGG AGA TGT GG- 3'; IFN- $\gamma$ forward primer 5'-CCA ACG CAA AGC AAT ACA TGA-3', reverse primer 5'-CGC TTC CCT GTT TTA GCT GC-3'; IL-1 $\beta$ forward primer 5'TTG AAG AAG AGC CCA TCC TC-3', reverse primer 5'-CAG CTC ATA TGG GTC CGA C-3'; IL-6 forward primer 5'-CCG GAG AGG AGA CTT CAC-3', reverse primer 5'-TCC ACG ATT TCC CAG AGA-3'; occludin forward primer 5'-GGC AGC AGC TTG TTA AGC AG3', reverse primer 5'-ACT TGG CGC AGT GGT AAG CA-3', zonula occludens-1 (ZO-1) forward primer 5'-GCC GCT AAG AGC ACA GCA A-3', reverse primer 5'-TCC CCA CTC TGA AAA TGA GGA-3'; $\beta$-actin forward primer 5'-GAG ACC TTC AAC ACC CCA GC-3', reverse primer 5'-ATG TCA CGC ACG ATT TCC C-3'. The cycle-threshold $(\mathrm{Ct})$ value was automatically shown by the instrument's software, and the relative mRNA expression level of each gene was calculated using the $2^{-\Delta \Delta \mathrm{Ct}}$ method. The reference gene $\beta$-actin was used as a normalizer.

\section{Western Blot}

The fresh intestinal tissue was homogenized in radioimmunoprecipitation lysis buffer (RIPA buffer) with the protease inhibitor phenylmethane sulfonyl fluoride (PMSF, ThermoFisher scientific, 36978, IL, USA). Total protein concentration was measured using the BCA Protein Assay Kit (ThermoFisher Scientific, 23225, IL, USA) following the manufacturer's instructions; $20 \mu \mathrm{g}$ total protein was loaded per lane and separated by $10 \%$ sodium dodecylsulfate (SDS)-polyacrylamide gel electrophoresis, and then was transferred onto $0.45 \mu \mathrm{m}$ nitrocellulose (NC) membrane (0.45 $\mu \mathrm{m}$, Bio-Rad Laboratories, 1620115, Hercules, CA) according to the standard protocols. After blocking for 
$1 \mathrm{~h}$ with $5 \%$ milk in TBST buffer, membranes were incubated with rabbit anti-OZ 1 antibody (1:500, AF5145) or rabbit anti-occludin antibody $(1: 500,27260-1-\mathrm{AP})$ at $4^{\circ} \mathrm{C}$ overnight. After incubation with HRP Goat anti-Rabbit Ig (1:2000, Gab97200) for $2 \mathrm{~h}$ at room temperature, proteins were detected using enhanced chemiluminescence (ECL) reagent (Millipore, Billerica, MA).

\section{Lactulose/mannitol assay}

Intestinal permeability was evaluated using lactulose/mannitol assay ( $\mathrm{L} / \mathrm{M}$ assay), which was performed as previously described (26). Briefly, fasted rats were gavaged with the lactulose $(100 \mathrm{mg} / \mathrm{mL})$ and mannitol $(50 \mathrm{mg} / \mathrm{mL})$ solution and put into metabolic cages. Rat urine was collected during the following $8 \mathrm{~h}$. Then, the urine sample was centrifugated at 10,000 r/min for $10 \mathrm{~min}$ and added 5\% acetic acid. After that, the sample was boiled, centrifuged, and filtered to remove impurities. At last, the purified specimen was analyzed with high-performance liquid chromatography to calculate the ratio of lactulose and mannitol (L/M ratio). The change of intestinal permeability was indicated by the urine content of L/M leaked from the intestine.

\section{Analysis of gut microbiota}

The total genomic DNA of the fecal samples was extracted using a QIAamp DNA Stool Mini Kit; 16S rRNA gene V3-V4 regions were amplified with specific primers $343 \mathrm{~F}$ (5'-TACGGRAGGCAGCAG-3') and 798R (5'-AGGGTATCTAATCCT-3') following a previously described method (27). All PCRs were performed using a Phusion Flash High-Fidelity PCR Master Mix, and mixed PCR products in equal quantities were purified using a Gene JETTM Gel Extraction Kit. Sequencing libraries were acquired with an Ion Plus Fragment Library Kit. Lastly, the library was sequenced on an Ion S5 TM XL platform, and $400 \mathrm{bp} / 600 \mathrm{bp}$ single-end reads were generated (Novogene Co., Ltd. Beijing, China). After sequencing, the $16 \mathrm{~S}$ rRNA sequence data were analyzed using bioinformatics methods, as previously described (28). UPARSE software (Uparse v7.0.1001, http://drive5.com/uparse/) assigned the sequences with $\geq 97 \%$ similarity to the same operational taxonomic units (OTUs). The representative sequence of every OTU was annotated taxonomic information according to the Silva Database (Version 132, https://www.arbsilva.de/). The principal coordinate analysis (PCoA) and microbial diversity were performed via Novomagic (https://magic.novogene.com).

\section{Statistical analysis}

The results were expressed as the mean \pm standard deviation (SD). Body weight and food intake were analyzed using repeated-measures mixed models, assuming unstructured covariance. For other data, Student's $t$-test was used to compare differences between two groups, and a one-way analysis of variance (ANOVA), followed by a post hoc Tukey's test, was used to determine differences between more than two groups. The Spearman's correlation coefficient was calculated with GraphPad Prism 8 (La Jolla, CA, USA), and the clustering results were visualized with heatmaps constructed and analyzed using Cluster 3.0 and Java Treeview software 1.6. Values of $P<$ 0.05 were considered statistically significant.

\section{Results}

\section{Effects of CLA on fat mass and liver}

Currently, the commercially available CLA product is commonly synthesized from linoleic acid by alkaline isomerization (29). In this study, the crude CLA mixture was purified using the method of urea-inclusion crystallization (15). According to the GC analysis results in Fig. 1a and b, the purity of CLA increased from 70.21 to $94.68 \%$. The highly purified CLA included $35.84 \% \mathrm{c}-9$, t-11 CLA, and $56.25 \% \mathrm{t}-10$, c-12 CLA, which suggested that the function of CLA was more similar to that of t-10, c-12 CLA.

To study the effect of highly pure CLA on the food intake and body weight of $\mathrm{OB}$ mice, we recorded the food consumed in $24 \mathrm{~h}$ and body weight once per week. At the end of the 2 nd week, the average body weight of the $\mathrm{OB}$ group was significantly higher than that of the WT group (Fig. 1c). This showed that ob/ob mouse was an excellent genetically obese model to assess the effects of CLA on congenital obesity. After a 5-week treatment, body weight was noticeably decreased in both WT-CLA $(P<0.05)$ and OB-CLA $(P<0.01)$ groups (Fig. 1c), and the results of body weight gain showed the same trend (Fig. 1d). There was no significant difference in food intake between the WT and WT-CLA or OB and OB-CLA groups (Fig. 1e), which suggested that the effect of CLA on body weight might be due to increased fat metabolism, not appetite reduction. Lee's indexes for assessing obesity in the WT-CLA and OBCLA groups were significantly decreased compared with the WT $(P<0.05)$ and OB $(P<0.05)$ groups, respectively (Fig. 1f). These results demonstrated that CLA prevented body weight gain in both wild-type and ob/ob mice and was more effective on weight loss in the obese mice.

CLA supplementation induced a decrease in fat tissue mass in both the WT and OB mice (Fig. 2a). The weight of visceral and subcutaneous fat also showed dramatic effects of CLA (Fig. 2b and c). H\&E staining of fat tissue further confirmed the anti-obesity effect of CLA, with smaller adipocytes in the WT-CLA and OB-CLA groups than in the WT and OB groups separately (Fig. 2a and d).

We investigated the effect of CLA on hepatic steatosis, observing that $\mathrm{OB}$ mice had a larger liver than the WT mice. CLA treatment enlarged the liver of the WT mice but reduced the size of the liver in the OB mice (Fig. 2e). 
a

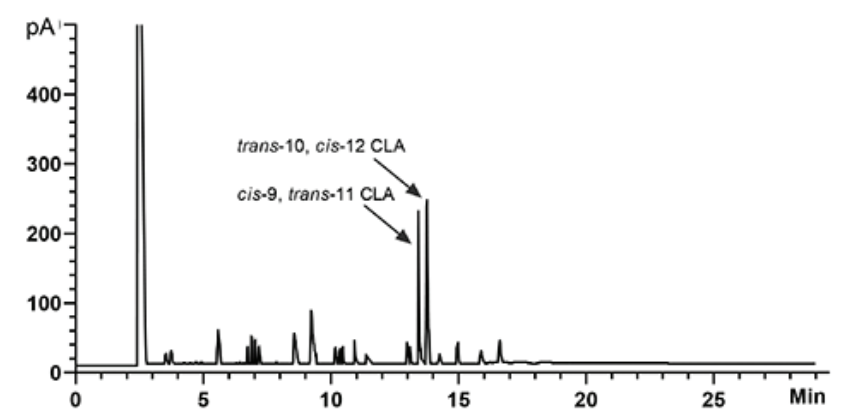

D

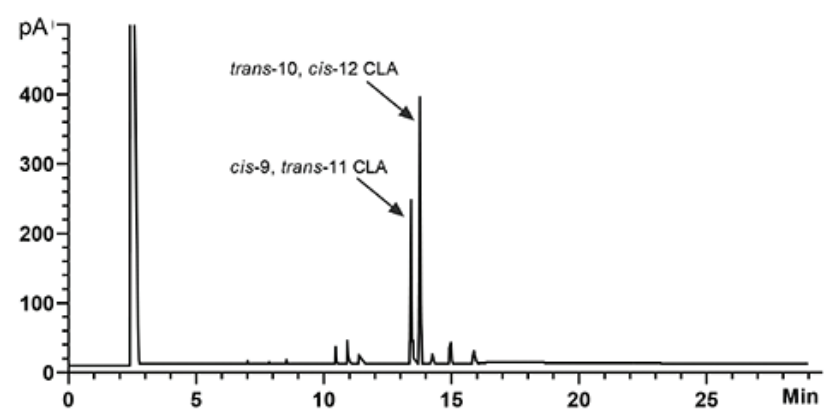

C
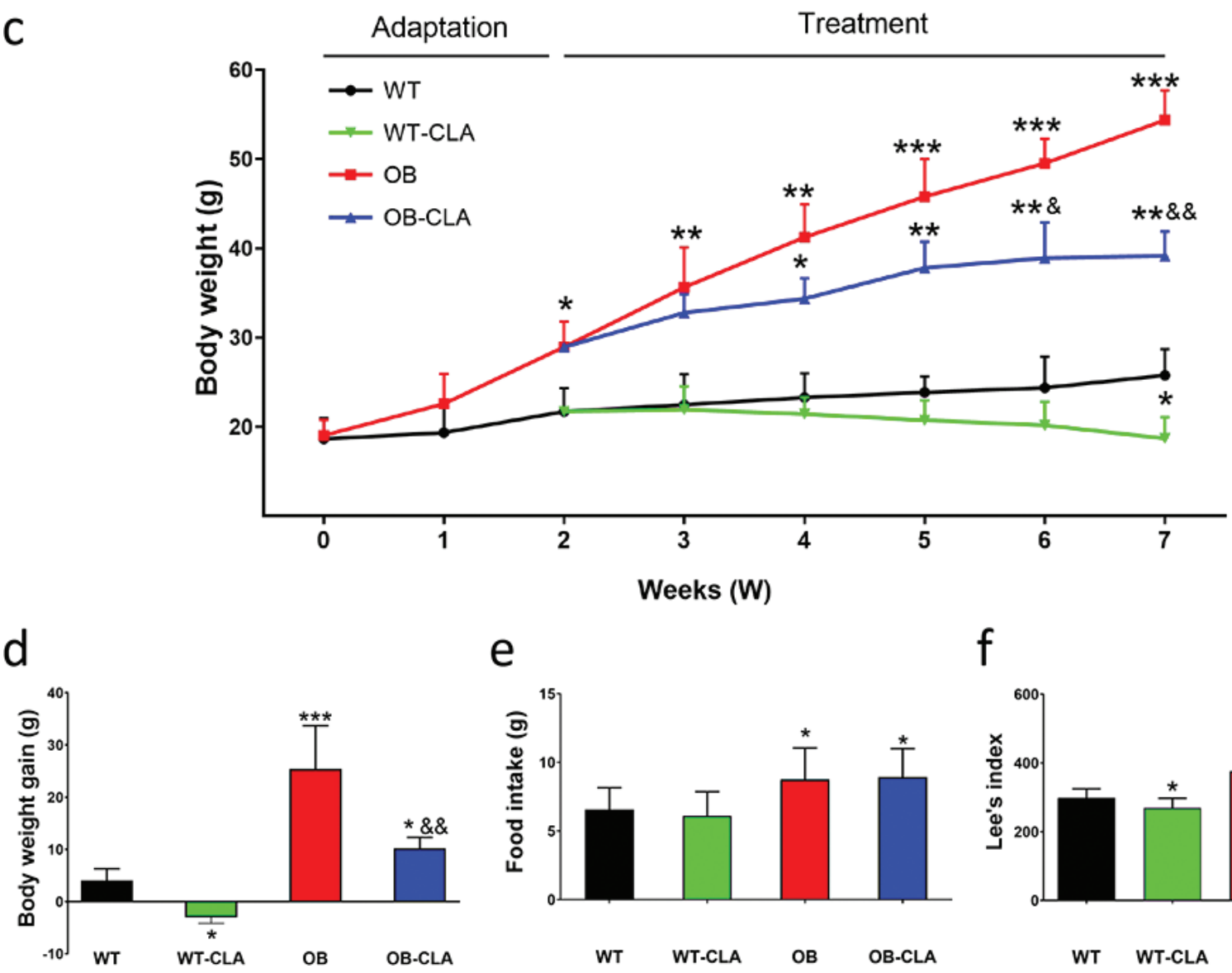

e

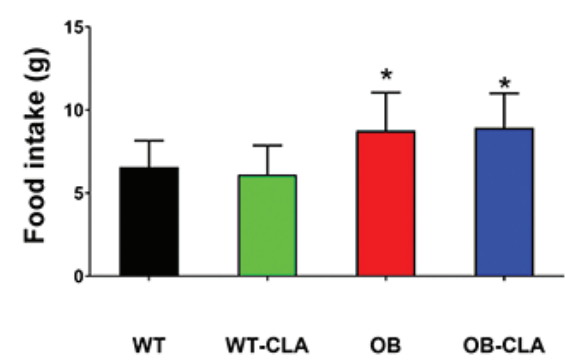

$f$

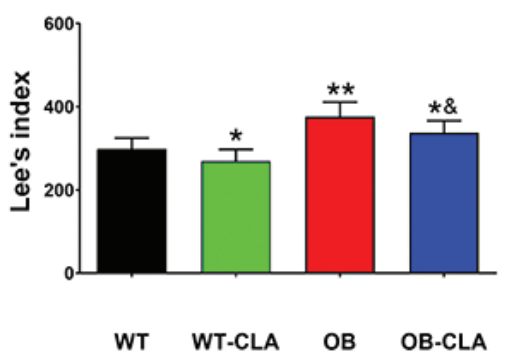

Fig. 1. Meteorological chromatograms and weight loss effects of CLA. The meteorological chromatograms of crude CLA (a) and highly pure CLA (b) were showed. The changes in body weight (c), body weight gain (d), food intake (e), and Lee's index (f) of mice during the 7-week CLA supplementation are displayed. The data are expressed as means \pm SD. Comparison with the WT group, ${ }^{*} P<0.05,{ }^{* *} P<0.01,{ }^{* * *} P<0.001$; comparison with the OB group, $\& P<0.05, \& \& P<0.01, \& \& \& P<0.001 ;(n=8)$.

The liver weight was significantly increased by CLA in the WT-CLA group compared with the WT group $(P<0.05)$ and was remarkably reduced in the OB-CLA mice $(P<$ 0.01 , Fig. $2 f$ ), which was consistent with the different hepatocyte sizes of the four groups, as shown by the H\&E staining (Fig. 2e and g). Therefore, CLA significantly decreased fat mass in both WT and OB mice but induced different effects of hepatic lipid accumulation in the WT and $\mathrm{OB}$ mice.
Effects of CLA on serum and liver lipids in $O B$ mice

The serum lipid concentrations of the OB group were significantly higher than those of the WT group (Fig. 3a-c), which suggested that the OB mice had hyperlipidemia. CLA treatment notably decreased the serum levels of TG, TC, and FFA of the OB-CLA group compared with those of the OB group (all $P<0.05$, Fig. 3a-c). For the WT mice, CLA showed few effects on serum lipids compared with the OB mice (Fig. $3 \mathrm{a}-\mathrm{c}$ ). 

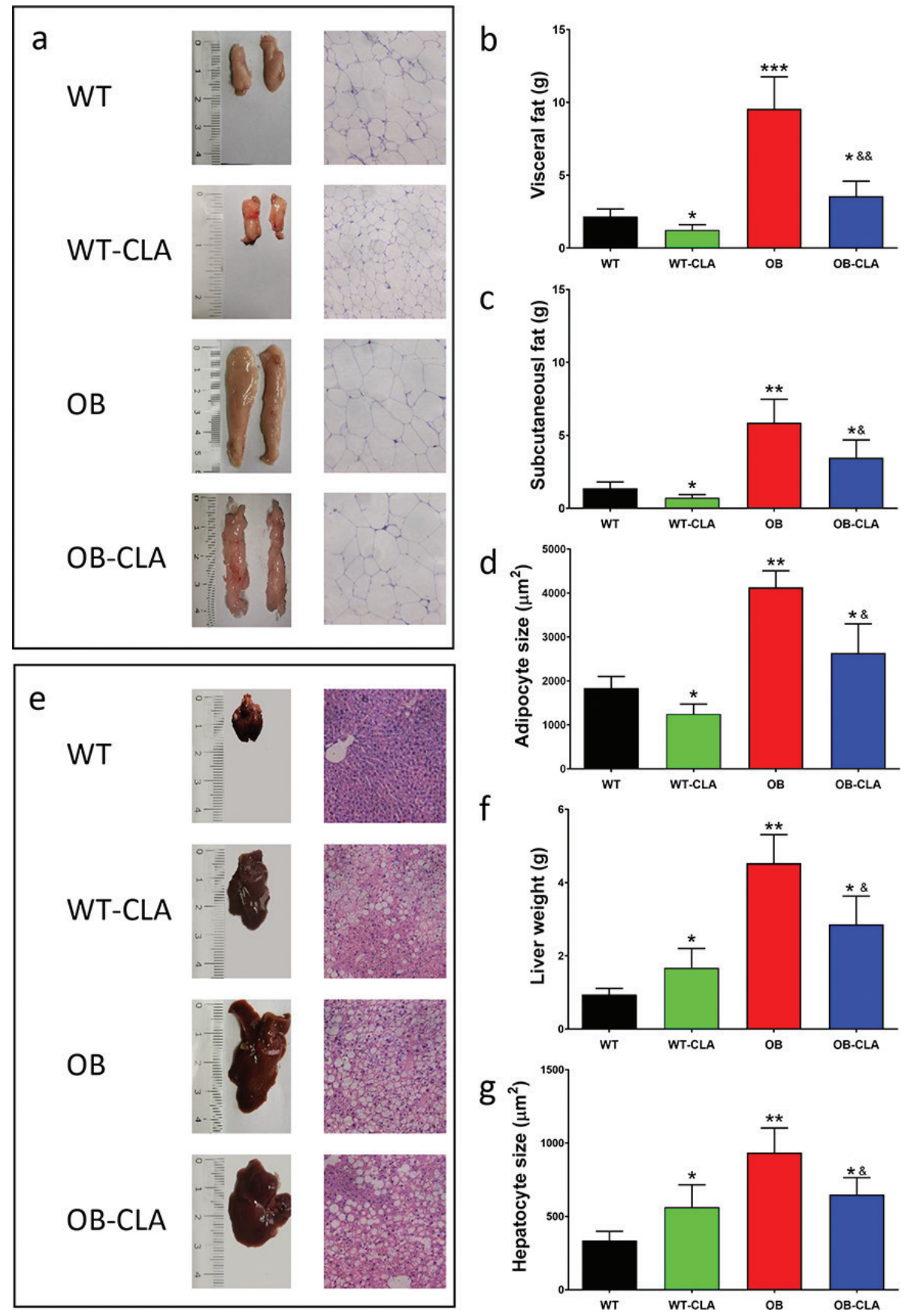

Fig. 2. Effects of CLA on fat tissue and liver. CLA supplementation significantly reduced the volume of fat mass (a), the weight of visceral fat (b), and subcutaneous fat (c) in the WT and OB mice. The H\&E staining of fat mass (a) and adipocyte size measurement (d) showed the effect of CLA on the adipocytes. The effects of CLA on the livers of WT and OB mice were shown: (e) hepatic morphology and H\&E staining, liver weight (f), and hepatocyte size (g). The data are expressed as means \pm SD. Comparison with the WT group, ${ }^{*} P<0.05,{ }^{* *} P<0.01,{ }^{* * *} P<0.001$; comparison with the OB group, $\& P<0.05, \& \& P<0.01 ;(n=8)$.

Liver lipids were extracted and analyzed, revealing that the amount of liver lipids in the WT-CLA group was significantly higher than that of the WT group $(P<0.05)$, while CLA markedly decreased the lipid content of the OB-CLA group compared with the OB group $(P<0.01$, Fig. 3d). The lipid composition of the livers is shown in Fig. 3e. CLA significantly increased the triacylglycerol content of the liver $(26.84 \pm 3.59 \%)$ in the WT-CLA group compared with the WT group $(20.54 \pm 4.78 \%$, $P<0.05)$, but the triacylglycerol was markedly decreased in the OB-CLA group $(31.28 \pm 3.19 \%)$, which was mainly balanced by the phospholipid content. The proportion of total cholesterol and free FAs in the liver was not affected by CLA in either the WT or OB mice. Table 1 presents the FA composition of the liver lipids. The proportions of long-chain saturated fatty acids (LCSFAs, 14:0, 16:0, 
a
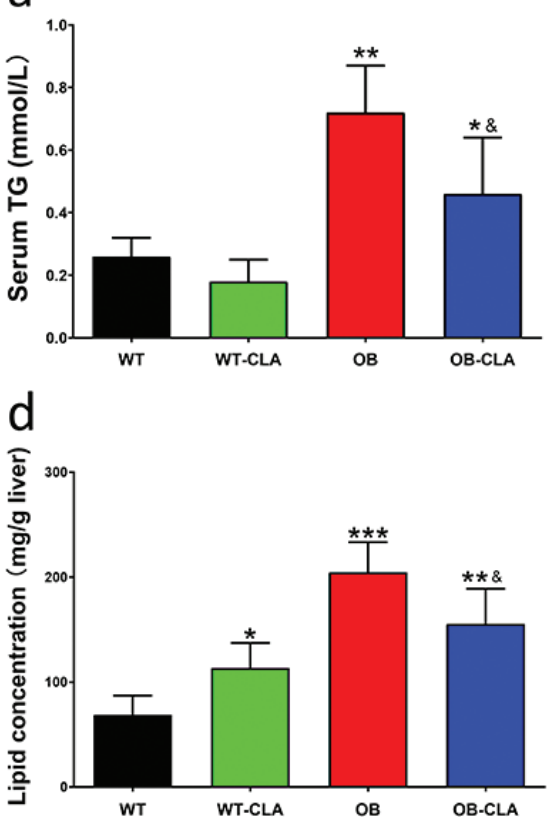

b

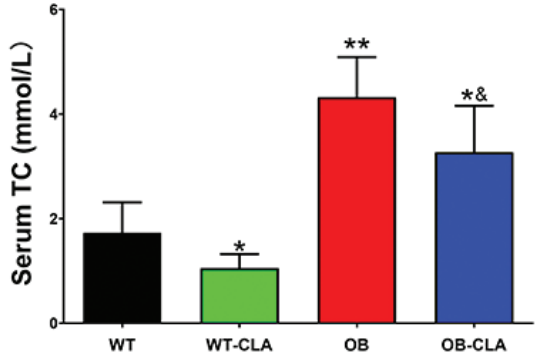

e
C

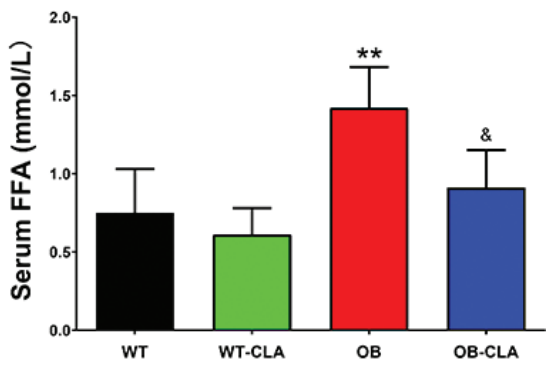

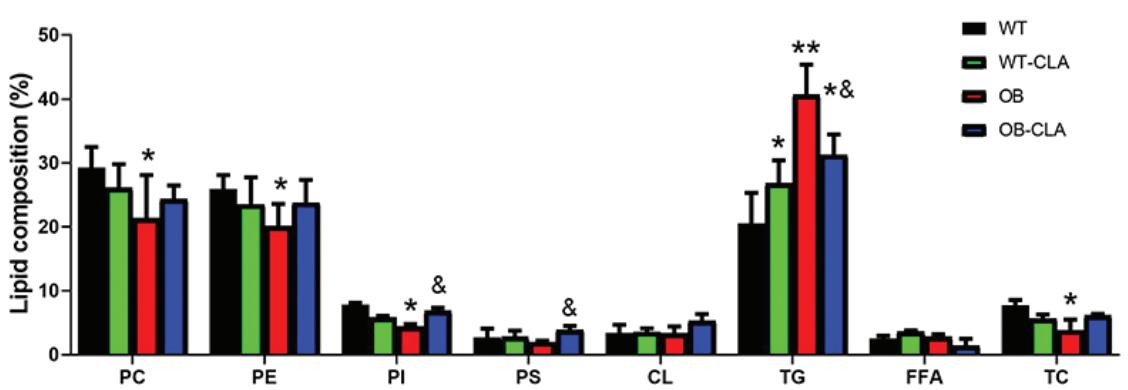

Fig. 3. Effects of CLA on serum lipid levels and liver lipid composition. Serum lipids were measured, including TG (a), TC (b), and FFA (c), and were decreased by CLA in OB mice. CLA increased the lipid concentration of the liver in the WT mice, but decreased the lipid concentration in the OB mice (d). CLA modified the lipid composition of the liver in the WT and OB mice (e). The data are expressed as mean $\pm \mathrm{SD}$. Comparison with the WT group, ${ }^{*} P<0.05, * * P<0.01, * * * P<0.001$. Comparison with the OB group, $\& P<0.05 ;(n=8)$.

Table 1. Long-chain fatty acid composition (\%) of liver lipids in the mice fed CLA

\begin{tabular}{|c|c|c|c|c|}
\hline Fatty acid & WT & WT-CLA & OB & OB-CLA \\
\hline 14:0 & $0.21 \pm 0.08$ & $0.48 \pm 0.25 *$ & $0.43 \pm 0.23$ & $0.27 \pm 0.15^{8}$ \\
\hline 16:0 & $22.01 \pm 3.38$ & $27.54 \pm 4.88 *$ & $29.01 \pm 6.1$ & $20.46 \pm 3.39^{\&}$ \\
\hline $16: 1$ & $2.39 \pm 0.5$ & $3.14 \pm 0.89$ & $4.14 \pm 0.51$ & $4.23 \pm 0.61$ \\
\hline 18:0 & $4.92 \pm 2.32$ & $6.63 \pm 2.68 *$ & $6.16 \pm 3.23$ & $3.85 \pm 0.39^{8}$ \\
\hline I8:। & $|3.88 \pm 3.6|$ & $14.18 \pm 3.24$ & $26.26 \pm 4.37$ & $18.59 \pm 3.65^{8}$ \\
\hline $18: 2 ; 9,12$ & $18.57 \pm 1.23$ & $14.56 \pm 2.27^{*}$ & $11.64 \pm 1.96$ & $15.84 \pm 2.97^{8}$ \\
\hline I 8:2 (9, I I-CLA) & $0.09 \pm 0.04$ & $1.05 \pm 0.19 * * * *$ & $0.02 \pm 0.02$ & $1.82 \pm 0.29^{828}$ \\
\hline $18: 2(10,12-C L A)$ & $0.02 \pm 0.01$ & $\mathrm{I} .44 \pm 0.24 * * *$ & $0.04 \pm 0.03$ & $2.38 \pm 0.05^{828}$ \\
\hline $20: 3$ & $2.38 \pm 0.25$ & $2.41 \pm 0.17$ & $2.52 \pm 0.18$ & $2.15 \pm 0.18$ \\
\hline $20: 4 ; 5,8, I I, I 4$ & $12.44 \pm 5.18$ & $5.01 \pm 3.25 *$ & $7.64 \pm 3.79$ & $13.74 \pm 3.02^{8}$ \\
\hline $22: 6$ & $13.08 \pm 3.18$ & $11.25 \pm 2.37$ & $10.37 \pm 4.8$ & $12.46 \pm 2.38$ \\
\hline
\end{tabular}

Comparison with the WT group, ${ }^{*} P<0.05$, $* * * P<0.001$. Comparison with the OB group, ${ }^{\circledR} P<0.05$, ${ }^{2 \& \&} P<0.001$.

and 18:0) were higher in the WT-CLA group compared with the WT group $(P \mathrm{~S}<0.05)$, but were decreased in OBCLA mice $\left(P_{\mathrm{S}}<0.05\right)$. The proportion of unsaturated FAs (e.g., 20:4;5,8,11,14 and 18:2;9,12) was significantly reduced by CLA in the WT mice $(P<0.05)$, while it was dramatically increased in the OB mice $(P<0.05)$. CLA administration significantly elevated the proportion of 18:2 $(9,11$ and 10,12) in the liver FA of both WT and OB mice $(P<0.0001)$. These data suggest that CLA modified the composition of liver lipids and FAs in WT and OB mice.
Effects of CLA on hepatic inflammatory cytokines and intestinal permeability

Inflammation is an important risk factor of hepatic steatosis, so the mRNA expression of several important inflammatory cytokines in the liver was examined. In the WT mice, CLA enhanced the gene expression of TNF- $\alpha$ and IL-6 in the liver $(P<0.05$, Fig. $4 \mathrm{a}$ and $\mathrm{d})$. The increased gene expression levels of $\mathrm{TNF}-\alpha, \mathrm{IFN}-\gamma$, and IL-1 $\beta$ in the OB mice were significantly inhibited in the OB-CLA group $(P<0.05$, Fig. $4 \mathrm{a}-\mathrm{c})$. The liver mainly receives venous blood from the intestine via the portal 


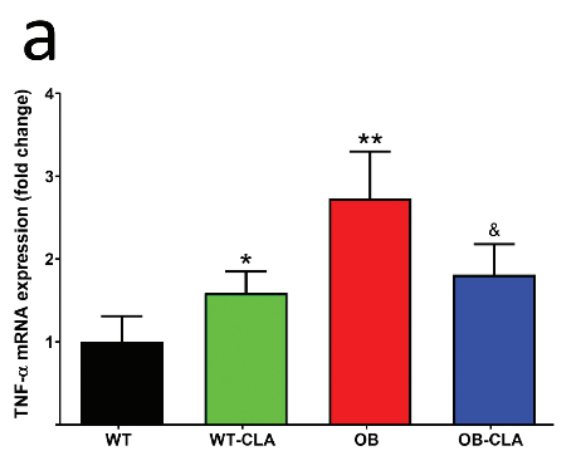

\section{b}
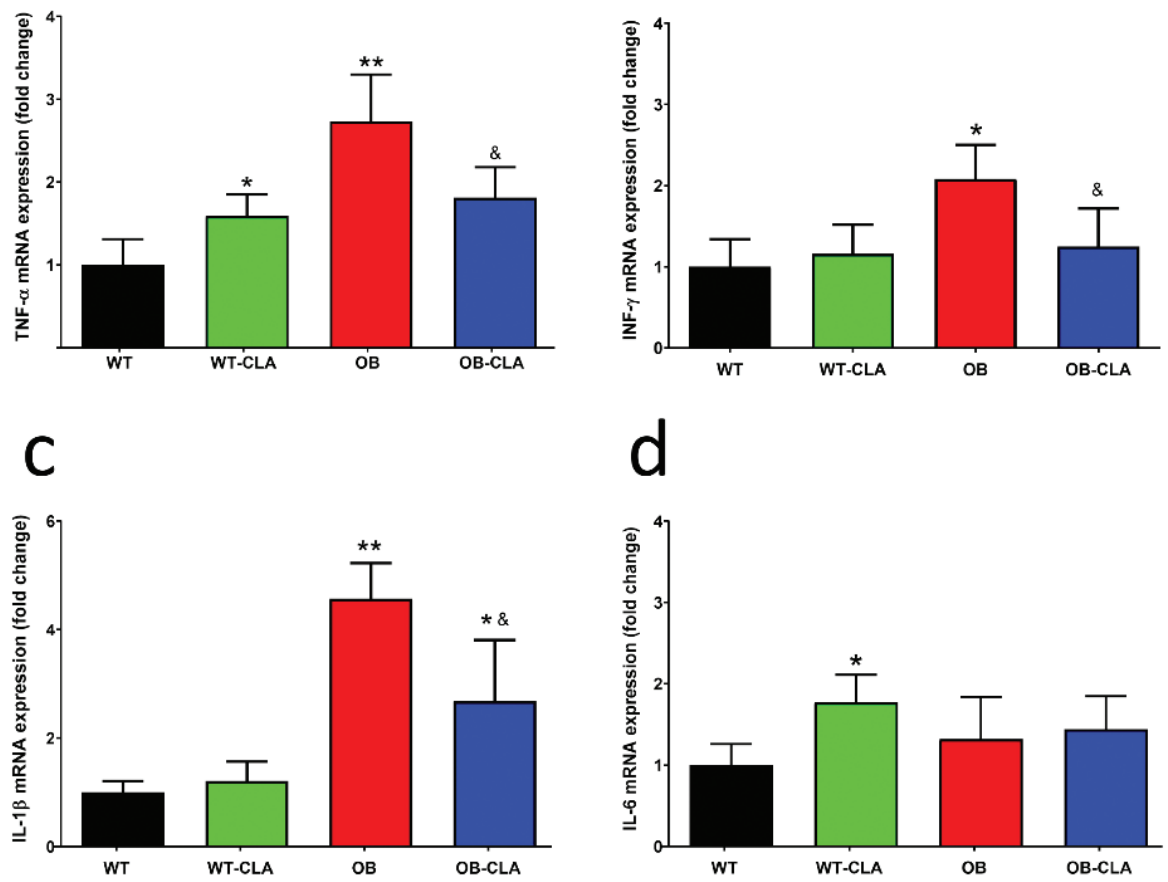

d
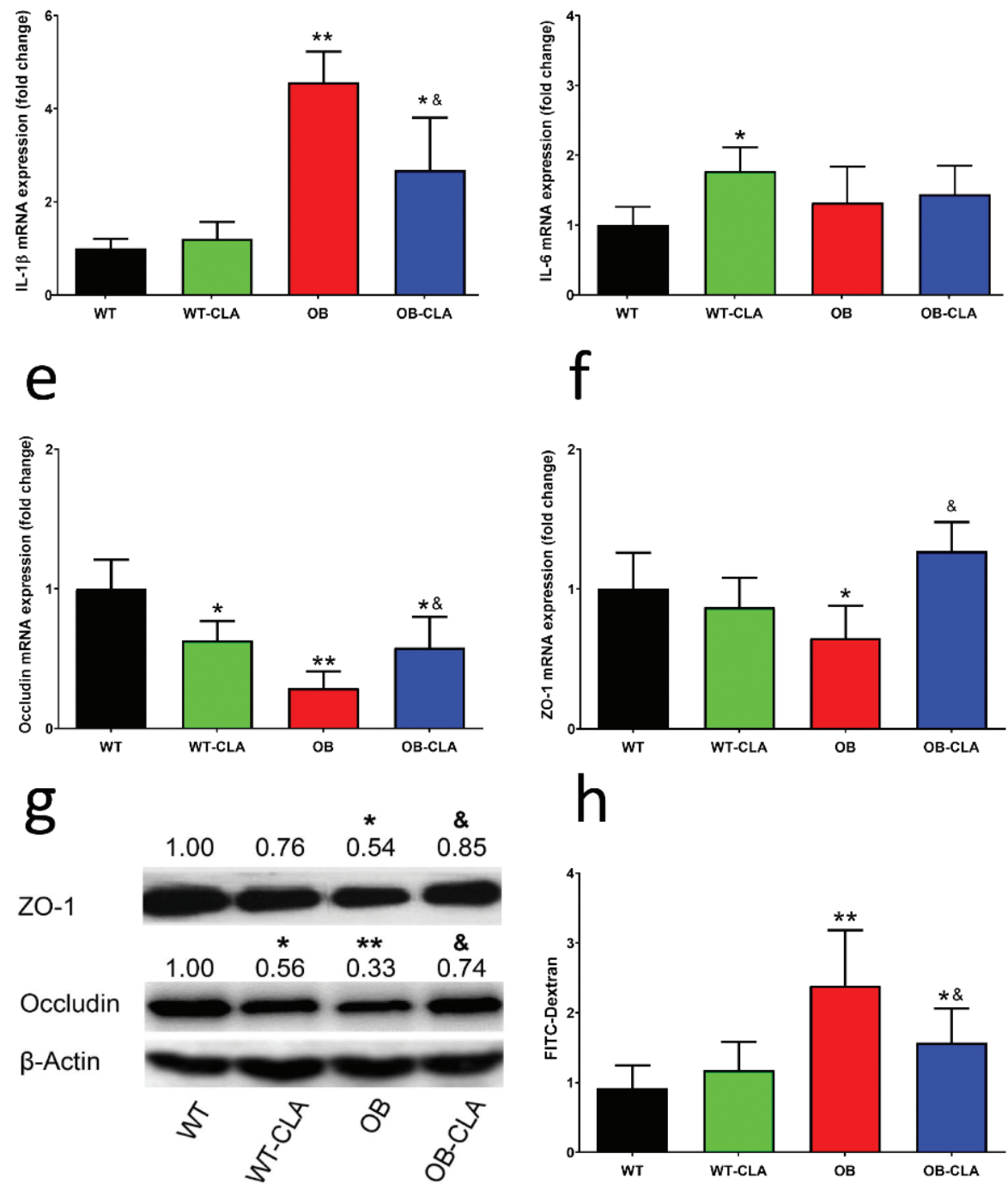

Fig. 4. Effects of CLA on the mRNA expression of inflammatory cytokines and intestinal permeability. CLA treatment influenced the mRNA-expressing levels of TNF- $\alpha$ (a), IFN- $\gamma$ (b), IL-1 $\beta$ (c), and IL-6 (d) in the liver. The mRNA expression levels of the intestinal tight junction proteins occludin (e) and ZO-1 (f) in colon tissue were measured using RT-qPCR. The protein expression levels of occludin and ZO-1 in colon tissue were detected with western blot (g). Lactulose/mannitol assay was used to check the intestinal permeability (h). The data are expressed as mean $\pm \mathrm{SD}$. Comparison with the WT group, $* P<0.05, * * P<$ 0.01 ; comparison with the $\mathrm{OB}$ group, $\& P<0.05 ;(n=8)$. 
vein, which contains nutrients and other substances that are absorbed in the intestines. Thus, hepatic inflammation might be related to intestinal permeability.

Intestinal permeability is closely associated with changes in tight-junction structural proteins (30), involving occludin and ZO-1. RT-PCR investigation revealed that the mRNA expressions of occludin and ZO-1 in the OB group were significantly lower than that of the WT group $(P<0.01$ and $P<0.05$, Fig. $4 \mathrm{e}$ and $\mathrm{f})$. CLA treatment decreased mRNA expressions of occludin in the proximal colon in the WT mice $(P<0.05$, Fig. $4 \mathrm{e})$, while it increased the lower occludin and ZO-1 mRNA level in the OB mice $(P<0.05$, Fig. 4 e and f). The protein expression of occludin and ZO-1 in the colon was further detected by western blot. The results showed that the $\mathrm{OB}$ mice had lower occludin and OZ-1 expression than that of the WT mice $(P<0.01$ and $P<0.05$, Fig. $4 \mathrm{~g})$, which was markedly restored by CLA treatment $(P<0.05$, Fig. $4 \mathrm{~g})$. While for the WT mice, CLA depressed the expression of occludin protein in the WT-CLA group $(P<0.05$ vs. WT group, Fig. 4g).

The lactulose/mannitol assay was used to assess intestinal permeability. As shown in Fig. 4h, the L/M ratio of OB group was significantly higher than that of the CON group $(P<0.01)$, indicating that the intestinal permeability was greatly increased in obesity mice. CLA treatment obviously decreased the L/M ratio of OB-CLA group $(P<0.05$ vs. OB group), but had little effect on the mice of CON-CLA group. It suggested that CLA may be able to reduce the damage of intestinal epithelial tight junctions induced by obesity, possibly changing the contents of the hepatic portal vein.

\section{Effects of CLA on gut microbiota}

Gut microbiota play important roles in lipid metabolism and absorption. Here, we collected feces samples to analyze the composition of gut microbiota with regard to CLA modulation. The OTUs were clustered with a standard of $97 \%$ similarity according to $16 \mathrm{~S}$ rRNA sequences, identifying 574 OTUs. To describe sample similarity and overlap, the number of common and unique OTUs is shown in a Venn diagram (Fig. 5a). The unique and total numbers of OTUs in the WT, WT-CLA, OB, and OBCLA groups were 74/990, 28/794, 28/934, and 30/896, respectively. Thus, the ratios of unique to total OTUs of each group were $7.47,3.53,3.00$, and $3.35 \%$, respectively. The PCoA of the weighted UniFrac distance displayed four completely separate clusters (Fig. 5b). The results revealed that the gut microbiota of the OB mice were dramatically different from that of the WT mice (PC1, $48.67 \%$ ). CLA treatment partly reversed the shift in gut microbiota in the OB mice (PC2, 21.83\%, Fig. 5b). Based on the clustering results, CLA supplementation altered the community of gut microbiota in the WT and OB mice and improved the diversity of gut microbiota to a normal level.

The effects of CLA on gut microbiota composition and the relative abundances of the bacterial species, especially dominant ones, at different levels of classification were assessed. At the phylum level, the three major phyla of gut microbiota in the WT group were Bacteroidetes, Firmicutes, and Proteobacteria. There were significant increases in the relative abundance of Firmicutes $(51.86 \%)$ in the OB group compared with the WT group (14.42\%, Fig. 5c). The ratio of Firmicutes/Bacteroidetes (F/B) of the OB group was notably higher than that of the WT group ( 1.2875 vs. $0.4296, P<0.01)$, which was decreased by CLA treatment in the OB-CLA group (1.0580, Fig. 5c). Definitely, the relative abundance of Proteobacteria was recovered in the OB-CLA group (11.78\%), which was remarkably decreased in the OB group (1.68\%).

The effect of CLA supplementation on the relative abundance of gut microbiota was also observed at the genus level. The top 35 most-abundant genera were presented in a heat map and were classified into four different clusters according to the change in different components (Fig. 5d). In cluster 1, the OB group showed higher levels of some pro-inflammatory bacteria (e.g., Enterorhabdus, Tyzzerella, Alistipes, and Rikenella), which might be high-risk pathogens that increase intestinal permeability (31-33). CLA supplementation significantly decreased the abundance of these bacteria in the OB-CLA group, suggesting that CLA probably reduced the reproduction of these pro-inflammatory bacteria. In cluster 2, the WT-CLA group had higher proportions of Muribaculum, Faecalibaculum, and Lactobacillus than the WT group. In cluster 3, CLA treatment significantly increased well-known beneficial bacteria, such as Lachnoclostridium, Roseburia, Dubosiella, Oscillibacter, and Anaerostipes, in the OB-CLA group. Cluster 4 showed that the proportions of Bacteroides, Odoribacter, Anaerotruncus, and Marvinbryantia in the WT-CLA group were significantly lower than that in the WT group, while CLA supplementation was not so effective in the OB mice.

For a clearer change in genus, the abundances of three representative bacteria were further analyzed. The typical pro-inflammatory bacteria, Tyzzerella and Alistipes, were more abundant in the OB group compared with the WT group, which was reversed in the OB-CLA group (Fig. 5e and f). Another beneficial bacterium, Anaerostipes, was obviously increased after CLA supplementation in the WT and OB mice (Fig. 5g).

\section{Correlation between gut microbiota and hepatic steatosis}

Spearman's correlation coefficient was used to analyze the connection between intestinal bacteria and hepatic steatosis. The correlation heat map (Fig. 6a) showed that the 
a

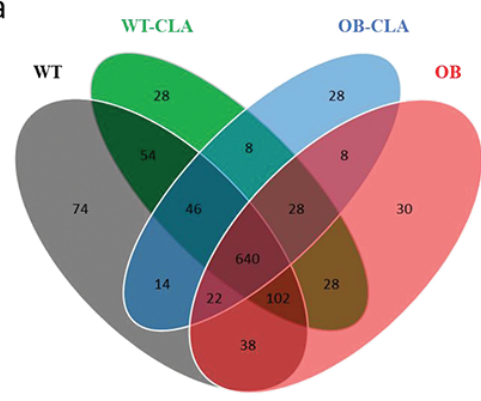

C
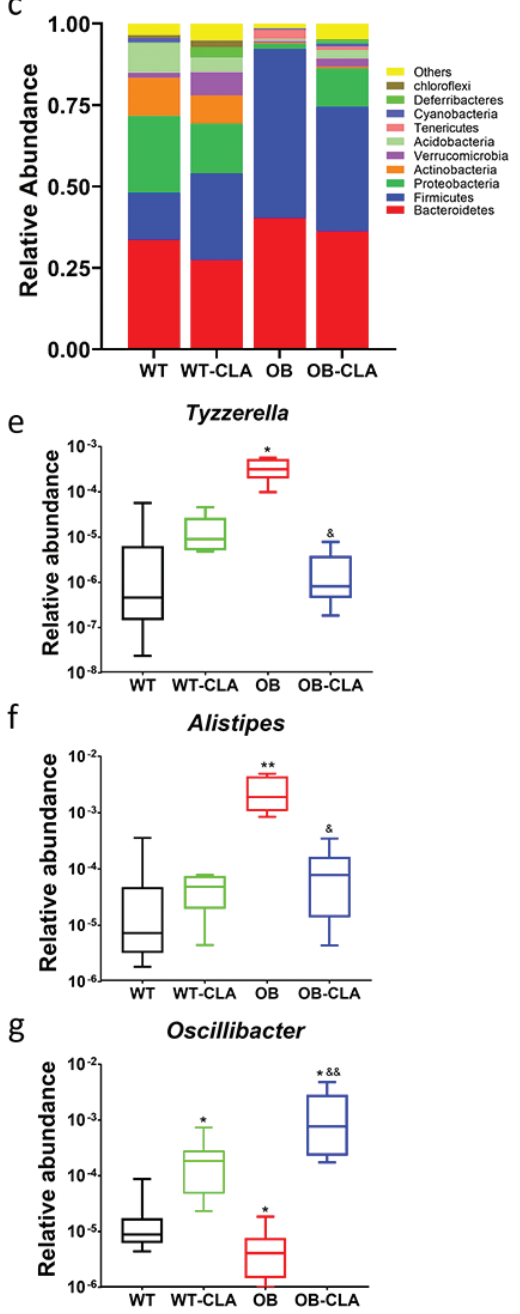

b
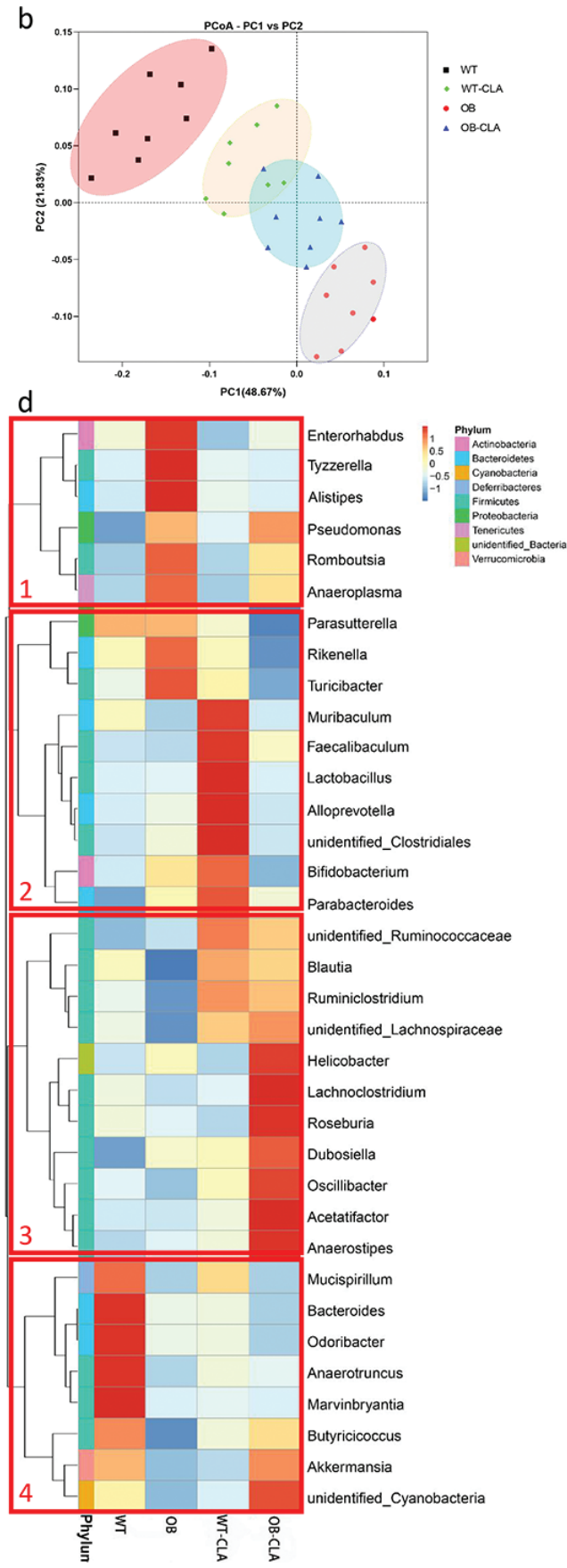

Fig. 5. Effects of CLA on the gut microbial community. Collected fecal samples were analyzed based on the 16S rRNA sequence V3-V4 regions, and the results were shown as follows: (a) OTU cluster analysis of the gut microbiota, (b) the modified gut microbial community after CLA treatment, (c) the 10 dominant microbial communities at the phylum level in different groups, and (d) the microbial community bar plot at the genus level. The relative abundances of three typical bacteria (Tyzzerella, Alistipes, and Oscillibacter) are displayed (e).

bacteria Tyzzerella, Alistipes, and Turicibacter had a widely positive correlation with liver lipids (TG and LCSFAs) and inflammation (gene expression of TNF- $\alpha$, IFN- $\gamma$, IL$1 \beta$, and IL-6), but displayed a negative relationship with the intestinal tight junction (gene expression of occludin and ZO-1). Ruminiclostridium, Muribaculum, and Blautia showed a strongly positive correlation with intestinal tight junction, and Ruminiclostridium was negatively correlated with liver lipids and inflammation. These results indicate that gut microbiota were closely associated with liver inflammation, lipid levels, and intestinal permeability, which might contribute to hepatic steatosis in the model mice. 


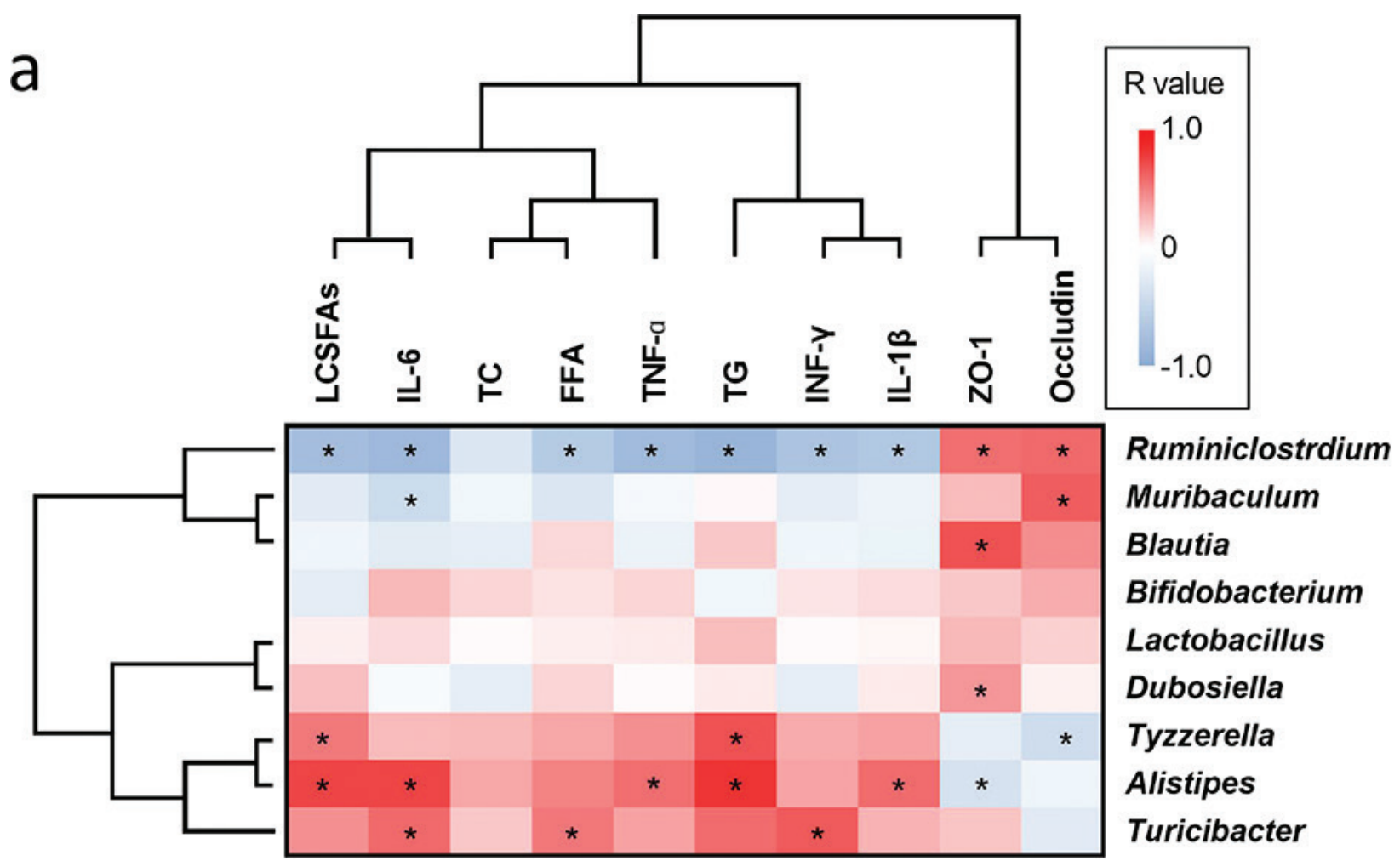

b

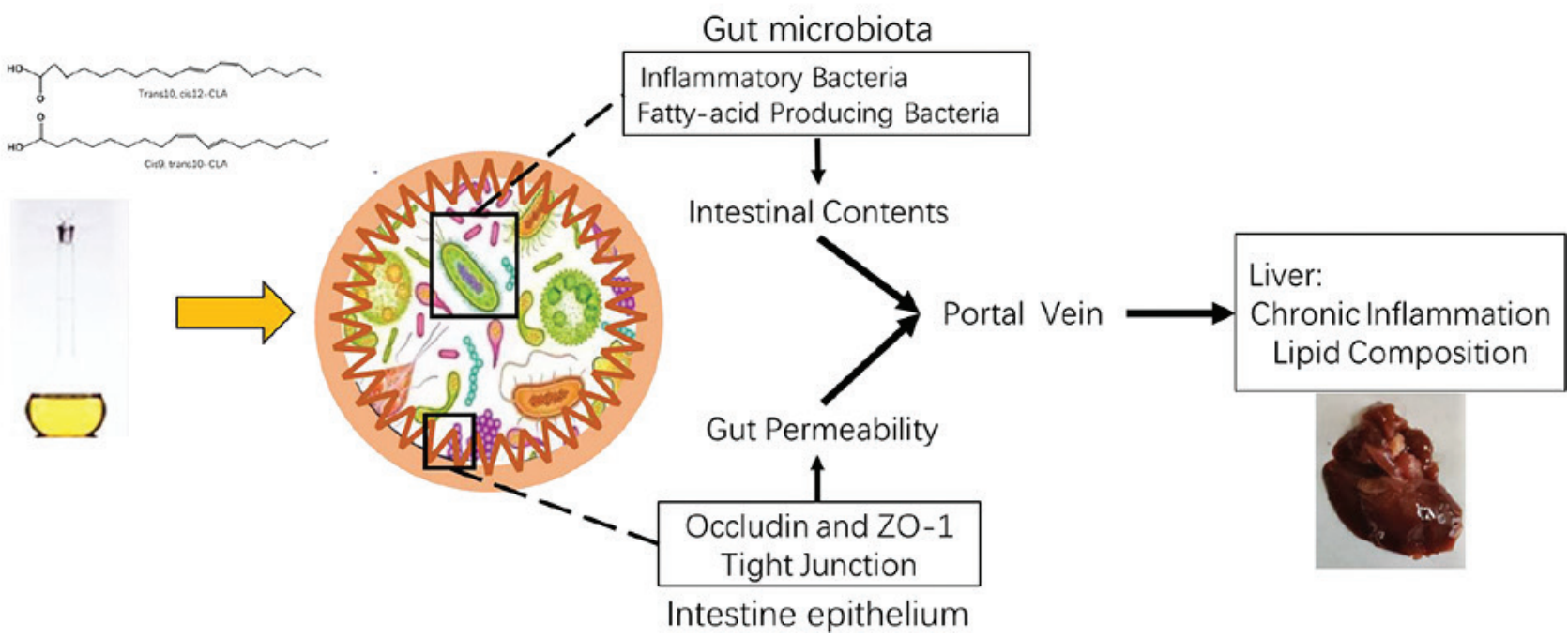

Fig. 6. Correlation analysis between physiological data and gut microbial composition at the genus level. (a) The association between physiological data and gut microbial composition was displayed in a heatmap. (b) The scheme describes how CLA affects hepatic steatosis via a multifactor pattern.

\section{Discussion}

In this study, highly purified CLA was prepared using urea-inclusion crystallization. The normal lipid accumulation in the liver cells of normal rats was increased by CLA supplementation in the WT-CLA group, while the excess hepatic lipid accumulation in the ob/ob mice was significantly decreased in the OB-CLA group. Although the size of hepatocytes in the OB-CLA group was still larger than that of the WT-CLA group, CLA potentially contributed to ameliorating the hepatic steatosis in obese mice. Liver lipid analysis showed that there were different lipid classes and FFA compositions in the WT-CLA and OB-CLA groups. CLA promoted hepatic inflammatory and intestinal permeability in the WT mice, but it 
decreased the mRNA expression of liver TNF- $\alpha$, IFN- $\gamma$, and IL-1 $\beta$ and improved the lower intestinal tight junction proteins in the OB mice. The gut microbiota test indicated a higher abundance of beneficial bacteria (e.g., Lachnoclostridium, Roseburia, Dubosiella, Oscillibacter, and Anaerostipes) and a lower abundance of pro-inflammatory bacteria (e.g., Tyzzerella and Alistipes) in the OB-CLA group compared with the OB group. Our correlation analysis suggested that the change in gut microbiota modified the hepatic FA composition and improved liver inflammation and intestinal permeability in OB mice.

Preclinical and human studies have suggested the beneficial effects of CLAs against cancer, obesity, and atherosclerosis (2). However, CLA has about 28 different isomers, and the two major isomers (cis-9, trans-11, and trans-10, cis-12) exhibited different fat-reducing properties. Thus, the purity and isomer ratio of CLA are key factors in determining its biological functions. Commercially available CLA products are commonly synthesized from linoleic acid (LA) by alkaline isomerization (29). In this study, urea-inclusion crystallization with a 2-step process was used to yield CLA with a purity of $94.68 \%$. Moreover, the purified CLA contained more trans-10 and cis-12 CLA isomers. CLA treatment resulted in an effective anti-obesity effect in $\mathrm{OB}$ mice, and this function was mainly exhibited by trans-10, cis-12 CLA $(2,34)$. Thus these results further confirmed that the type and ratio of CLA isomers were important to its biological function.

As with most studies on CLA, body weight, fat tissue mass, and serum lipid levels were significantly downregulated by highly purified CLA in WT and OB mice (2). However, the controversial topics were that CLA treatment induced hepatic steatosis in the WT mice $(3,4)$ and reversed the obesity-induced hepatic lipid accumulation in the OB mice (8), which indicated that the effect of CLA on the liver was related to body conditions. Although some studies have suggested that decreased leptin and subsequent insulin resistance might be the reasons for hepatic steatosis induced by CLA (5-7), leptin deficiency in the ob/ob mice did not aggravate lipid accumulation in the OB-CLA group, which was similar to a previous study (8).

In this study, liver lipid analysis showed that there were more saturated FAs in the WT-CLA group and more unsaturated FAs in the OB-CLA group. Martha et al. found that in mice with normal food, CLA increased 14:0, 16:0, and 18:0 FA composition of the liver and reduced arachidonate $(20: 4 ; 5,8,11,14)(35)$. In obese rats with a highfat diet, CLA supplementation induced an increase in the polyunsaturated FAs in the liver lipids (36). The data suggest that CLA affected hepatic lipid metabolism in these mice. Liang et al. reported that liver inflammation causes dysfunction of hepatic lipid metabolism in rodents (37). Our results also showed that CLA treatment modified liver inflammation by increasing the mRNA expression of
TNF- $\alpha$ and IL- 6 in the WT mice with lipid accumulation, while reducing TNF- $\alpha$, IFN- $\gamma$, and IL- $1 \beta$ in the OB mice. The liver has a dual blood supply from the hepatic arteries and portal vein. Since serum lipid levels were decreased by CLA in WT mice, the main source of lipids accumulated in the liver might has originated from the portal vein. It is well known that the portal vein transports substances absorbed from the stomach and intestine; thus, we further tested intestinal permeability and gut microbiota.

Previous animal experiments suggest that higher intestinal permeability and subsequent intraportal LPS infusion are major mechanisms of fatty liver disease (38). Antje et al. also confirmed that the extent of liver steatosis in humans is related to intestinal permeability (39). This permeability of the intestine depends on tight junctions composed of specialized proteins, such as occludin, ZO-1, claudins, and junctional adhesion molecules (40). In this study, CLA decreased the mRNA and protein expressions of occludin of the proximal colon in WT mice, which may weaken intercellular adhesion and increase permeability. In the OB mice, CLA increased the lower mRNA and protein expression of occludin and ZO-1 to recover intestinal permeability. Combined with the results of liver inflammation, we speculate that higher intestinal permeability in the WT-CLA group was accompanied by an increased liver inflammation response, and low-grade liver inflammation was observed in the OB-CLA group with recovered intestinal permeability. Therefore, the different intestinal permeability between the CLA-treated and untreated groups might be an important factor influencing CLA-induced hepatic steatosis.

The gut microbiota have been found to play a major role in the weight loss induced by CLA. Den Hartigh et al. reported that weight loss and divergent metabolic effects of mice fed t10c12-CLA or food restriction were associated with compositional differences in the gut microbiota, including an abundance of Allobaculum, Butyrivibrio, and Bifidobacteria with t10c12-CLA and enrichment of Bacteroides by FR (41). In this study, CLA treatment significantly changed the abundance and composition of gut microbiota. At the bacterial genus level, the WT-CLA group had higher proportions of Lactobacillus, Muribaculum, and Faecalibaculum than the WT group. Lactobacillus is a type of SLCFA-producing bacteria (42); thus, the higher composition of saturated FAs in the livers of the WT-CLA group might have originated from the intestine. Muribaculum and Faecalibaculum have been reported to be pro-inflammatory and anti-inflammatory genera, respectively (43, 44 ), and they might be related to the elevation of mRNA expression of TNF- $\alpha$ and IL- 6 in the WT-CLA group. Tyzzerella and Alistipes, the typical pro-inflammatory bacteria (45), were abundant in the OB group, which improved the opportunity to induce the hyperinflammatory 
state of the liver via portal vein. In the OB-CLA group, CLA not only decreased the abundance of these pro-inflammatory bacteria but also significantly increased the abundance of Lachnoclostridium, Roseburia, Dubosiella, Oscillibacter, and Anaerostipes, which are believed to be beneficial for health for their anti-inflammatory and anti-oxidative stress activities, short-chain FA production, polyunsaturated FA transport, and other properties (46-48). The altered abundances of bacterial genera in the different groups were statistically related to intestinal permeability, liver inflammation, and hepatic lipid levels, suggesting that the hepatic steatosis induced by CLA is complex and multifactorial.

In conclusion, the findings from this study suggest a novel mechanism of hepatic lipid accumulation induced by CLA by influencing intestinal barrier function and gut microbial distribution (Fig. 6b). Therefore, understanding and applying highly purified CLA may be beneficial in promoting its anti-obesity and fatty liver-preventing effects.

\section{Acknowledgments}

This work was supported by the National Key Research and Development Program of China (2018YFD0901103), the China Ocean Mineral Resources Research and Development Association (DY135-B2-14, DY135-E2-1), the China Postdoctoral Science Foundation (2020M671999), the National Key Research and Development Program of China (2018YFD0901105), the National Natural Science Foundation of China (32000074, 42176130, 31971060), the Natural Science Foundation of Shandong Province (ZR202102190351, ZR2019BD023), the Medical and Health Science and Technology Development Project of Shandong Province (2018WS378), the Tai Mountain Industry Leading Talent of Shandong (2019TSCYCX-06), the Science and Technology Planning Project of Guangxi (AA21196002).

\section{Conflict of interest and funding}

The authors have no conflicts of interest to declare that are relevant to the content of this article. The authors have not received any funding or benefits from the industry or elsewhere to conduct this study.

\section{References}

1. Zabala A, Portillo MP, Macarulla MT, Rodriguez VM, Fernandez-Quintela A. Effects of cis-9, trans-11 and trans-10, cis-12 CLA isomers on liver and adipose tissue fatty acid profile in hamsters. Lipids 2006; 41(11): 993-1001. doi: 10.1007/ s11745-006-5050-5

2. den Hartigh LJ. Conjugated linoleic acid effects on cancer, obesity, and atherosclerosis: a review of pre-clinical and human trials with current perspectives. Nutrients 2019; 11(2): 370. doi: $10.3390 /$ nu11020370
3. den Hartigh LJ, Wang S, Goodspeed L, Wietecha T, Houston B, Omer M, et al. Metabolically distinct weight loss by 10,12 CLA and caloric restriction highlight the importance of subcutaneous white adipose tissue for glucose homeostasis in mice. PLoS One 2017; 12(2): e0172912. doi: 10.1371/journal.pone.0172912

4. Bezan PN, Holland H, de Castro GS, Cardoso JFR, Ovidio PP, Calder PC, et al. High dose of a conjugated linoleic acid mixture increases insulin resistance in rats fed either a low fat or a high fat diet. Exp Clin Endocrinol Diabetes 2018; 126(6): 379-86 doi: 10.1055/s-0043-118348

5. Fu C, Zhang Y, Yao Q, Wei X, Shi T, Yan P, et al. Maternal conjugated linoleic acid alters hepatic lipid metabolism via the AMPK signaling pathway in chick embryos. Poult Sci 2020; 99(1): 224-34. doi: 10.3382/ps/pez462

6. Vyas D, Kadegowda AK, Erdman RA. Dietary conjugated linoleic Acid and hepatic steatosis: species-specific effects on liver and adipose lipid metabolism and gene expression. J Nutr Metab 2012; 2012: 932928. doi: 10.1155/2012/932928

7. Stout MB, Liu LF, Belury MA. Hepatic steatosis by dietaryconjugated linoleic acid is accompanied by accumulation of diacylglycerol and increased membrane-associated protein kinase C epsilon in mice. Mol Nutr Food Res 2011; 55(7): 1010-7. doi: 10.1002/mnfr.201000413

8. Wendel AA, Purushotham A, Liu LF, Belury MA. Conjugated linoleic acid fails to worsen insulin resistance but induces hepatic steatosis in the presence of leptin in ob/ob mice. J Lipid Res 2008; 49(1): 98-106. doi: 10.1194/jlr.M700195-JLR200

9. Albillos A, de Gottardi A, Rescigno M. The gut-liver axis in liver disease: pathophysiological basis for therapy. J Hepatol 2020; 72(3): 558-77. doi: 10.1016/j.jhep.2019.10.003

10. Duan Y, Llorente C, Lang S, Brandl K, Chu H, Jiang L, et al. Bacteriophage targeting of gut bacterium attenuates alcoholic liver disease. Nature 2019; 575(7783): 505-511. doi: 10.1038/ s41586-019-1742-x

11. Todoric J, Di Caro G, Reibe S, Henstridge DC, Green CR, Vrbanac A, et al. Fructose stimulated de novo lipogenesis is promoted by inflammation. Nat Metab 2020; 2(10): 1034-45. doi: 10.1038/s42255-020-0261-2

12. Barron LK, Bao JW, Aladegbami BG, Colasanti JJ, Guo J, Erwin CR, et al. Toll-like receptor 4 is critical for the development of resection-associated hepatic steatosis. J Pediatr Surg 2017; 52(6): 1014-19. doi: 10.1016/j.jpedsurg.2017.03.026

13. Han Y-H, Onufer EJ, Huang L-H, Sprung RW, Davidson WS, Czepielewski RS, et al. Enterically derived high-density lipoprotein restrains liver injury through the portal vein. Science 2021; 373(6553). doi: 10.1126/science.abe6729

14. Chen Y, Yang B, Ross RP, Jin Y, Stanton C, Zhao J, et al. Orally administered CLA ameliorates DSS-induced colitis in mice via intestinal barrier improvement, oxidative stress reduction, and inflammatory cytokine and gut microbiota modulation. J Agric Food Chem 2019; 67(48): 13282-98. doi: 10.1021/acs.jafc.9b05744

15. Kim YJ, Lee KW, Lee S, Kim H, Lee HJ. The production of high-purity conjugated linoleic acid (CLA) using two-step urea-inclusion crystallization I and hydrophilic arginine-CLA complex. J Food Sci 2003; 68(6): 1948-51. doi: 10.1111/j.13652621.2003.tb06999.x

16. Aldai N, Delmonte P, Alves SP, Bessa RJB, Kramer JKG. Evidence for the initial steps of DHA biohydrogenation by mixed ruminal microorganisms from sheep involves formation of conjugated fatty acids. J Agric Food Chem 2018; 66(4): 842-55. doi: 10.1021/acs.jafc. 7 b04563 
17. Banni S, Carta G, Angioni E, Murru E, Scanu P, Melis MP, et al. Distribution of conjugated linoleic acid and metabolites in different lipid fractions in the rat liver. J Lipid Res 2001; 42(7): 1056-61. doi: 10.1016/S0022-2275(20)31594-7

18. Lei F, Zhang XN, Wang W, Xing DM, Xie WD, Su H, et al. Evidence of anti-obesity effects of the pomegranate leaf extract in high-fat diet induced obese mice. Int J Obes (Lond) 2007; 31(6): 1023-9. doi: 10.1038/sj.ijo.0803502

19. Devaligoda GKYP, Hemantha S, Tharanga T. Determination of adipocyte cell size by $\mathrm{H} \& \mathrm{E}$ stained adipose tissue and collagenase digested isolated adipocytes. Curr Trends Biotechnol Pharm 2018; 12(2): 139-46.

20. Jin T, Jiang Z, Luan X, Qu Z, Guo F, Gao S, et al. Exogenous orexin-A microinjected into central nucleus of the amygdala modulates feeding and gastric motility in rats. Front Neurosci. 2020; 14: 274. doi: 10.3389/fnins.2020.00274

21. Parlee SD, Lentz SI, Mori H, MacDougald OA. Quantifying size and number of adipocytes in adipose tissue. Methods Enzymol 2014; 537: 93-122. doi: 10.1016/ B978-0-12-411619-1.00006-9

22. Nahar A, Baker AL, Nichols DS, Bowman JP, Britz ML. Application of thin-layer chromatography-flame ionization detection (TLC-FID) to total lipid quantitation in mycolic-acid synthesizing Rhodococcus and Williamsia species. Int J Mol Sci 2020; 21(5): 1670. doi: 10.3390/ijms21051670

23. Pang LQ, Liang QL, Wang YM, Ping L, Luo GA. Simultaneous determination and quantification of seven major phospholipid classes in human blood using normal-phase liquid chromatography coupled with electrospray mass spectrometry and the application in diabetes nephropathy. J Chromatogr B Anal Technol Biomed Life Sci 2008; 869(1-2): 118-25. doi: 10.1016/j. jchromb.2008.05.027

24. Sebedio JL, Juaneda P, Dobson G, Ramilison I, Martin JC, Chardigny JM, et al. Metabolites of conjugated isomers of linoleic acid (CLA) in the rat. Biochim Biophys Acta. 1997; 1345(1): 5-10. doi: 10.1016/s0005-2760(97)00015-5

25. Guo F, Xu L, Gao S, Sun X, Zhang N, Gong Y. Effect of orexin-A in the arcuate nucleus on cisplatin-induced gastric side effects in rats. Neurosci Res 2019; 143: 53-60. doi: 10.1016/j. neures.2018.06.001

26. Jarvinen KM, Konstantinou GN, Pilapil M, Arrieta MC, Noone S, Sampson HA, et al. Intestinal permeability in children with food allergy on specific elimination diets. Pediatr Allergy Immunol 2013; 24(6): 589-95. doi: 10.1111/pai.12106

27. Yang B, Chen HQ, Gao H, Wang JT, Stanton C, Ross RP, et al. Bifidobacterium breve CCFM683 could ameliorate DSS-induced colitis in mice primarily via conjugated linoleic acid production and gut microbiota modulation. J Funct Foods 2018; 49: 61-72. doi: 10.1016/j.jff.2018.08.014

28. Ma H, Zhang B, Hu Y, Wang J, Liu J, Qin R, et al. Correlation analysis of intestinal redox state with the gut microbiota reveals the positive intervention of tea polyphenols on hyperlipidemia in high fat diet fed mice. J Agric Food Chem 2019; 67(26): 7325-35. doi: 10.1021/acs.jafc.9b02211

29. Kim JH, Kim YJ, Park Y. Conjugated linoleic acid and postmenopausal women's health. J Food Sci 2015; 80(6): R1137-43. doi: 10.1111/1750-3841.12905

30. Han X, Fink MP, Yang R, Delude RL. Increased iNOS activity is essential for intestinal epithelial tight junction dysfunction in endotoxemic mice. Shock 2004; 21(3): 261-70. doi: 10.1097/01. shk.0000112346.38599.10

31. Wiersema ML, Koester LR, Schmitz-Esser S, Koltes DA. Comparison of intestinal permeability, morphology, and ileal microbial communities of commercial hens housed in conventional cages and cage-free housing systems. Poult Sci 2021; 100(2): 1178-91. doi: 10.1016/j.psj.2020.10.052

32. Rincel M, Olier M, Minni A, Monchaux de Oliveira C, Matime Y, Gaultier E, et al. Pharmacological restoration of gut barrier function in stressed neonates partially reverses long-term alterations associated with maternal separation. Psychopharmacology (Berl). 2019; 236(5): 1583-96. doi: 10.1007/s00213-019-05252-w

33. Ascher S, Reinhardt C. The gut microbiota: an emerging risk factor for cardiovascular and cerebrovascular disease. Eur J Immunol 2018; 48(4): 564-75. doi: 10.1002/eji.201646879

34. Marques TM, Wall R, O’Sullivan O, Fitzgerald GF, Shanahan F, Quigley EM, et al. Dietary trans-10, cis-12-conjugated linoleic acid alters fatty acid metabolism and microbiota composition in mice. Br J Nutr 2015; 113(5): 728-38. doi: 10.1017/ S0007114514004206

35. Belury MA, Kempa-Steczko A. Conjugated linoleic acid modulates hepatic lipid composition in mice. Lipids 1997; 32(2): 199-204. doi: 10.1007/s11745-997-0025-0

36. Sebedio JL, Angioni E, Chardigny JM, Gregoire S, Juaneda P, Berdeaux $\mathrm{O}$. The effect of conjugated linoleic acid isomers on fatty acid profiles of liver and adipose tissues and their conversion to isomers of $16: 2$ and 18:3 conjugated fatty acids in rats. Lipids 2001; 36(6): 575-82. doi: 10.1007/s11745-001-0759-8

37. Liang J, Chen S, Hu Y, Yang Y, Yuan J, Wu Y, et al. Protective roles and mechanisms of Dendrobium officinal polysaccharides on secondary liver injury in acute colitis. Int $\mathbf{J}$ Biol Macromol 2018; 107(Pt B): 2201-10. doi: 10.1016/j. ijbiomac.2017.10.085

38. Lam YY, Ha CW, Campbell CR, Mitchell AJ, Dinudom A, Oscarsson $\mathrm{J}$, et al. Increased gut permeability and microbiota change associate with mesenteric fat inflammation and metabolic dysfunction in diet-induced obese mice. PLoS One 2012; 7(3): e34233. doi: 10.1371/journal.pone.0034233

39. Damms-Machado A, Louis S, Schnitzer A, Volynets V, Rings A, Basrai M, et al. Gut permeability is related to body weight, fatty liver disease, and insulin resistance in obese individuals undergoing weight reduction. Am J Clin Nutr 2017; 105(1): 127-35. doi: 10.3945/ajen.116.131110

40. Li B, Lee C, Chuslip S, Lee D, Biouss G, Wu R, et al. Intestinal epithelial tight junctions and permeability can be rescued through the regulation of endoplasmic reticulum stress by amniotic fluid stem cells during necrotizing enterocolitis. FASEB J 2021; 35(1): e21265. doi: 10.1096/fj.202001426R

41. den Hartigh LJ, Gao Z, Goodspeed L, Wang S, Das AK, Burant $\mathrm{CF}$, et al. Obese mice losing weight due to trans-10, cis12 conjugated linoleic acid supplementation or food restriction harbor distinct gut microbiota. J Nutr 2018; 148(4): 562-72. doi: 10.1093/jn/nxy011

42. Zhao L, Huang Y, Lu L, Yang W, Huang T, Lin Z, et al. Saturated long-chain fatty acid-producing bacteria contribute to enhanced colonic motility in rats. Microbiome 2018; 6(1): 107. doi: 10.1186/s40168-018-0492-6

43. Reinoso Webb C, den Bakker H, Koboziev I, Jones-Hall Y, Rao Kottapalli K, Ostanin D, et al., Differential susceptibility to T cell-induced colitis in mice: role of the intestinal microbiota. Inflamm Bowel Dis 2018; 24(2): 361-79. doi: 10.1093/ibd/izx014

44. Benitez-Paez A, Gomez Del Pugar EM, Lopez-Almela I, Moya-Perez A, Codoner-Franch P, Sanz Y. Depletion of Blautia species in the microbiota of obese children relates to intestinal inflammation and metabolic phenotype worsening. mSystems 2020; 5(2): e00857-19. doi: 10.1128/ mSystems.00857-19 
45. Parker BJ, Wearsch PA, Veloo ACM, Rodriguez-Palacios A. The genus alistipes: gut bacteria with emerging implications to inflammation, cancer, and mental health. Front Immunol 2020; 11: 906. doi: 10.3389/fimmu.2020.00906

46. Louis P, Hold GL, Flint HJ. The gut microbiota, bacterial metabolites and colorectal cancer. Nat Rev Microbiol 2014; 12(10): 661-72. doi: 10.1038/nrmicro3344

47. Yan F, Li N, Shi J, Li H, Yue Y, Jiao W, et al. Lactobacillus acidophilus alleviates type 2 diabetes by regulating hepatic glucose, lipid metabolism and gut microbiota in mice. Food Funct 2019; 10(9): 5804-15. doi: 10.1039/c9fo01062a

48. Zeng Q, Li D, He Y, Li Y, Yang Z, Zhao X, et al. Discrepant gut microbiota markers for the classification of obesity-related metabolic abnormalities. Sci Rep 2019; 9(1): 13424. doi: 10.1038/ s41598-019-49462-w

\section{* Jinlai Miao}

Key Laboratory of Marine Eco-Environmental Science and Technology, First Institute of Oceanography, Ministry of Natural Resource, Qingdao, China; No.6 Xianxialing Road, Laoshan District, Qingdao, Shandong, China.

Email: miaojinlai@fio.org.cn 